\title{
Der Mensch im Spannungsfeld zwischen Heimatverlust, Heimatlosigkeit, Heimweh und Heimatsuche Psychoanalytische und psychosoziale Aspekte
}

\author{
Thomas Auchter (Aachen)
}

Zusammenfassung: Nach einer Begriffsklärung von Heimat werden entwicklungspsychologische Aspekte der Heimatbildung untersucht. Aktuelle Befunde von Entheimatung und Heimatverlust und ihre psychosozialen Folgen werden diskutiert. Der Psycho-sozio-pathologie von Heimat kann nur durch die Förderung einer stabilen inneren Heimat begegnet werden. Sie erlaubt eine Toleranz für Differenz.

Schlüsselwörter: Innere Heimat, Äussere Heimat, Heimatvertreibung, Fernweh, Rechtspopulismus

\section{$1 \quad$ Einführung}

Seit geraumer Zeit hat der Begriff «Heimat» (wieder) Konjunktur. Das ist zum einen bedingt durch die vielfältigen globalen Fluchtbewegungen und Migrationsprobleme. Für den Heimatverlust brauchen wir aber gar nicht in ferne Länder zu schauen, auch Braunkohlebagger schaffen z. B. in Nordrheinwestfalen oder in der Lausitz unwiederbringliches Verschwinden von Heimat!

Zum anderen hat der Begriff Aktualität durch das Erstarken von rechtspopulistischen Bewegungen überall auf der Welt, die diese Vorstellung für sich okkupieren und instrumentalisieren. Sie betrachten Heimat als Festung, die gegen andere abgeschottet wird durch Ideologien, Propaganda, Exklusion und Mauern. Sie schliessen damit an die missbräuchliche Verwendung des Heimatbegriffs durch die Nationalsozialisten an: «Heim ins Reich». Die Heimattümelei im Deutschland der Nachkriegszeit mit den verbreiteten Heimatliedern, Heimatromanen und Heimatfilmen hatte vor allem die Funktion, den Blick von der Scham und der Schuld über die deutschen Verbrechen der Nazizeit und des Zweiten Weltkrieges abzulenken (vgl. Schmoll, 2016, S. 22; Gross, 2019, S. 51 ff.). Seinerzeit bestand rund ein Viertel der Bevölkerung in Deutschland aus Heimatvertriebenen, Flücht- 
lingen, Migranten und «Displaced Persons» (Pavlović, 2016, S. 156). Die Jahrestreffen der Heimatvertriebenenverbände mit ihren Trachten, Liedern und Reden spekulierten lange Zeit über eine Rückkehr in die Heimat - was nur über eine Heimatvertreibung der nun dort Lebenden möglich gewesen wäre (vgl. Gross, 2019, S. 45f.). Erst die «Ostverträge» (1970) setzten dem ein Ende. Wobei zweifellos die «Heimatvertreibung» - egal wem und egal wo sie passiert - eine traumatische Erfahrung mit gravierenden seelischen Folgen darstellt (Langendorf, 2004; Varvin, 2016).

Derzeit ist die Thematik «Heimat» durch die Corona-Pandemie und ihre Folgen (verstärkte Fixierung auf das Zuhause und auf «Heimaturlaub») weiter aktualisiert.

"Home is where we start from", lautet der Titel eines posthum veröffentlichten Essay-Bandes von Donald W. Winnicott (1986). Im besten Fall beginnt also unser Lebensweg in einem Zuhause oder einer Heimat. Zugleich aber gilt: "Paradise lost'? In diesem Sinne ist Heimat etwas Verlorenes, das hinter uns liegt. Da dieses paradiesische Gefühl aber zumeist als etwas Gutes empfunden oder fantasiert wird, erwächst daraus Heimat als «Sehnsuchtsort», der vor uns liegt. Heimat hat ebenso mit Herkunft zu tun wie mit Ankunft (vgl. Gross, 2019, S. 19 ff.).

Das Bild einer Reise mit unaufhörlichem Aufbrechen und Ankommen ist eine treffliche Metapher für das menschliche Leben. Insofern ist all unser Ankommen im Leben immer nur vorläufig. «Wer jetzt kein Haus hat, baut sich keines mehr. Wer jetzt allein ist, wird es lange bleiben», beschreibt Rainer Maria Rilke (1902) in seinem Gedicht Herbsttag mögliche Probleme dieser Reise. Dazu gehören Unbehaustheit und Obdachlosigkeit als Varianten von Heimatlosigkeit. Umgekehrt preist Hermann Hesse (1941) in seinem Gedicht Stufen das Aufbrechen mit den Worten: «Und jedem Anfang wohnt ein Zauber inne, der uns beschützt und der uns hilft zu leben», sowie: «Wohlan denn, Herz, nimm Abschied und gesunde». Ähnlich formuliert auch der Text von Georg Thurmair, übrigens 1935 als verdecktes «AntiNazi-Lied» konzipiert: «Wir sind nur Gast auf Erden / und wandern ohne Ruh / mit mancherlei Beschwerden / der ewigen Heimat zu». Unsere Lebensreise endet soweit wir wissen - mit unserem Tod.

\section{Zur Begriffsbildung: «Heimat»}

Das Konzept«Heimat» ist ebenso komplex wie widersprüchlich (vgl. Gross, 2019). Diesem Facettenreichtum kann man im Grunde nur mit einer multidisziplinären Betrachtung gerecht werden. Im Bewusstsein der Begrenztheit beschränke ich mich im Wesentlichen auf die psychologische (psychoanalytische) und sozialpsychologische Perspektive. 
Im Begriff Heimat steckt das Wort «Heim» und es ist verwandt mit Haus, Zuhause, Daheim, also positiv konnotiert. Dazu gehört aber auch Heim wie Kinderheim, Altenheim, die zumindest zwiespältig, wenn nicht negativ konnotiert sind. Auch der Ausdruck «Heim-suchung» hat eine deutlich negative Bedeutung, noch mehr: «heim-tückisch». Nach vielfacher Aussage ist das deutsche Wort«Heimat» in andere Sprachen nicht übersetzbar.

Heimat kann sowohl mit einer äusseren wie mit einer inneren Realität (Auchter, 2013) in Verbindung gebracht werden. Heimat wird einerseits auf etwas Äusserliches bezogen, ein Dorf, eine Stadt, ein Land, eine Region oder eine Landschaft (territoriale Ebene). Heimat wird auch verbunden mit Herkunft und Zugehörigkeit z. B. zu einerVolksgruppe oder Religionsgemeinschaft (soziale oder kulturelle Ebene). Andererseits ist Heimat eine psychische Struktur, die vor allem etwas mit Emotionen und Sehnsüchten zu tun hat, insbesondere mit Sicherheit und Geborgenheit (emotionale Ebene).

Diese innere Heimat kann z. B. durch die Familie oder eine bestimmte soziale Gruppe fundamentiert werden. Christian Morgenstern (1922, S. 122) formulierte dazu: «Nicht da ist man daheim, wo man seinen Wohnsitz hat, sondern wo man verstanden wird». Das Konstrukt «Innere Heimat» resultiert aus haltvermittelnden (Auchter, 2000) und versichernden Beziehungsszenen zwischen Selbst und Objekten, die kognitiv und emotional internalisiert werden.

Der Begriff Heimat wurde bis ins 13. Jahrhundert nur für die jenseitige (himmlische) Heimat verwandt und galt nicht für irdische Angelegenheiten (Hoven-Buchholz, 2016, S. 142). Das mitteldeutsche Wort «heimōt» benannte zunächst das Heim, das Anwesen, den Hof (Schmoll, 2016, S. 20). Heimat hiess in der Schweiz und in Bayern ursprünglich soviel wie «Besitz» (des bäuerlichen Hofes) (Erdheim, 2016, S. 109).

Auch die Sprache (vgl. Gross, 2019, S.112ff.) oder der Dialekt können Heimatgefühle auslösen. So lautete das Karnevalsmotto 2019 in Köln: «Uns Sproch es Heimat»! Jean Améry (1988, S. 66 ff.), von den Nazis verfolgter und vertriebener jüdischer Schriftsteller, macht auf die enge Verbindung von Heimat und «Muttersprache» aufmerksam (vgl. auch Gross, 2019, S.112ff.). Insofern Heimat etwas mit frühkindlichen Erfahrungen mit Mutter und Vater zu tun hat, liegt die Verbindung zu «Mutterland» und «Vaterland» nahe. Sie werden synonym mit «Heimatland»verwendet. 


\section{$3 \quad$ Psychoanalyse und Heimat}

«Das Thema Heimatlosigkeit, Vertreibung und Exil ist zentral für die Geschichte der Psychoanalyse» (Leszczynska-Koenen, 2016, S. 214). Es begann damit, dass Sigmund Freud «seine Heimat als Arzt im medizinischen Denksystem verliess auf der Suche nach neuen Konzepten zum Verstehen der menschlichen Psyche und ihrer Erkrankungen» (Pavlović 2016, S. 155). 1938 wurde er wie die meisten jüdischen Psychoanalytiker von den Nazis aus seiner Heimatstadt Wien nach London vertrieben.

Im Registerband der Gesammelten Werke von Sigmund Freud taucht der Begriff «Heimat» nicht auf. Im Gesamttext seiner Schriften findet man ihn nur ein einziges Mal, nämlich als er das «weibliche Genitale» als «Eingang zur alten Heimat des Menschenkindes» bezeichnet (Freud, 1919h, S. 259).

Psychoanalytiker benötigen wie alle Menschen einen festen Boden unter ihren Füssen, eine geistige Heimat. Das bieten ihnen zum Ersten das psychoanalytische Theoriegebäude und zum Zweiten die psychoanalytischen Institutionen. Die Psychoanalyse eignet sich aber weder als Kathedrale noch als feste Burg. Auch wenn es zu allen Zeiten Psychoanalytiker gegeben hat, die ihre Zugehörigkeit zu einer psychoanalytischen Gruppe verabsolutiert haben und andere als «nichtpsychoanalytisch» ausgegrenzt und diffamiert haben. Insofern ist die Geschichte der Psychoanalyse auch eine Geschichte von «Spaltungen» (Hermanns, 1995). Adäquat ist eher ein offenes Haus mit einem stabilen Fundament, aber flexibel ausbaufähig mit «Übergangs-» und «Möglichkeitsräumen» (Winnicott; vgl. Auchter, 2004; 2013).

Der Psychoanalytiker steht auch vor der Notwendigkeit - vor allem in der konkreten Arbeit mit seinen Patienten - seine "psychoanalytische Heimat», sein theoretisches und praktisches Wissen «immer wieder aufzugeben und in Frage zu stellen» (Pavlović, 2016, S. 168)! Sich also dem Nicht-Wissen im Bezug auf den spezifischen Patienten und die spezifische therapeutische Beziehungssituation auszusetzen («gleichschwebende Aufmerksamkeit»)!

Vor vielen Jahrzehnten schenkte mir eine junge Patientin am Ende ihres längeren psychotherapeutischen Entwicklungsprozesses ein Blatt mit dem Foto eines alten, selbstzusammengeschusterten, einfachen Gewächshauses auf einem Acker. Darunter hatte sie das folgende selbstgestaltete Gedicht geschrieben:

\section{Gewächshaus:}

In Freiburgs lautester Strasse

Eine schwere, grosse Türe aus Holz, eine breite Treppe immer sauber, 
ein kahles, steriles Wartezimmer meist leer.

Ein Mensch holt mich ab,

führt mich hinein, schliesst Türen und Fenster,

setzt sich und schweigt.

Im Gewächshaus, dem Möglichkeitsraum einer Psychotherapie können Patient*-innen sich mit Heimatverlust, aber auch mit dem Schaffen «neuer Heimaten» befassen (vgl. Gross, 2019, S. 150, S. $158 \mathrm{ff}$.$) .$

\section{Aspekte von Heimat}

\subsection{Urheimat}

Von den meisten psychoanalytischen Autoren wird der neunmonatige Aufenthalt im Mutterleib als «Urheimat» (Janus, 2016, S. 73), als «früheste und erste Heimat» (a.a. O., S. 96), als «alte Heimat» (Freud 1919h, S. 259) oder explizit als «Mutterleibsheimat» (Messer, 2016, S. 100) betrachtet. Das Leben beginnt in einem Binnenraum.

Im besten Fall - aber nur dann! - stellt das «Mutterhaus» für die ersten intrauterinen neun Monate einen Raum des weitgehenden Geschützt- und Geborgenseins, der Sicherheit, des umfassenden Versorgung- und Befriedigungfindens dar. Da, wie schon Freud (1930a, S. 426) vermerkte, nichts im seelischen Leben verloren geht, bleiben aus dieser Zeit unbewusste seelische Spuren vor allem im Körpergedächtnis. Der Mutterleib ist allerdings nicht immer und nicht in jedem Fall eine paradiesische, vorambivalente, ideale Idylle und sicher nicht mehr am Ende der neun Monate, wo es in diesem ersten Zuhause ziemlich eng wird. Die intrauterine Erfahrung konfrontiert gleich mit den zwei wesentlichen Aspekten des Raumes, nämlich der Geborgenheit einerseits und der Bedrohung durch das Eingesperrtsein darin andererseits. Irgendwann kommt der Zeitpunkt, an dem die «Ur-höhle» zur «Ur-hölle» werden kann (vgl. deMause, 2000, zit. n. Funke, 2006, S. $169 \mathrm{ff}$.). Dann müssen wir aufbrechen, werden geboren und unsere Reise setzt sich ausserhalb vom «Hotel Mama» fort.

\subsection{Geburt-Exodus-"Paradise lost"}

Ähnlich wie das Intrauterinstadium eine ambivalente Raumerfahrung darstellt, lässt sich auch der komplexe Geburtsvorgang unter ganz unterschiedlichen Gesichtspunkten betrachten.

Otto Rank (1924) hat in seinem grundlegenden Werk: «Das Trauma der Geburt» die Geburt als «Urtrauma» und als «Urform der Angst» beschrie- 
ben (ebenso Freud, 1926d), also als Prototyp des Heimatverlusts. Für Donald W. Winnicott (1988) ist dagegen die Geburt vor allem der erste schöpferische Akt des Menschen, Ausdruck seiner eigenen Bewegungsimpulse, der sogenannten «spontanen Gesten», und eigenerWirkmächtigkeit und damit eine äusserst wertvolle (Winnicott, 1988, S. 144), kreative Erfahrung. Diese beiden Perspektiven schliessen sich keineswegs aus!

Die Geburt stellt die «Urform des Exodus» dar. Es ist die erste markante Erfahrung einer Trennung einer zuvor weitgehend als Einheit, respektive Ununterschiedenheit erlebten beziehungsweise fantasierten Situation. Michael Balint (1966, S. 83) hat diesen Ur-Zustand einmal mit dem schönen Bild der «Harmonie mit dem Grenzenlosen» gezeichnet. Romain Rolland und Sigmund Freud (1930a, S. 422) versuchen ihn mit dem Begriff «ozeanisches Gefühl» zu fassen.

Nach dem Geborenwerden zerbricht die Harmonie mit dem Grenzenlosen. Vorher «Selbstverständliches» muss nun vom Säugling aktiv in die eigene Hand genommen werden wie Atmen, Nahrung Aufnehmen und Ausscheiden.

Wenn das kleine Kind nach der Geburt in sein Leben aufbricht, ist zunächst einmal das meiste fremd und macht ihm Angst. Der «Neu-angst» steht jedoch die angeborene «Neu-gier» des Kindes entgegen. Der sichere (Rück-)Halt, den die Mutter und andere ihm durch ihre anhaltende liebevolle Präsenz bieten, die sichere «Bindung» (Bowlby), ermutigt das neugierige Explorationsverhalten des Kindes auch durch die Möglichkeit des «emotionalen Auftankens» (Mahler u.a., 1978, S. 91). Als biologischer «Nesthocker» ist das Neugeborene angewiesen auf ein «gutes Nest», in dessen Schutz, Geborgenheit und Sicherheit Entwicklung möglich wird, also eine «Beziehungsheimat».

\subsection{Nach der Geburt-Bedürfnis nach Beheimatung}

Heimat bleibt nach der Geburt lebenslang unbewusst auch ein Sehnsuchtsort der Rückkehr in den Mutterleib (Chasseguet-Smirgel, 1988), ins verlorene Paradies, gewissermassen Aufbrechen nach hinten, Heim-kehr. Aus der Vertreibung aus dem Paradies resultiert ein tiefes (regressives) Bedürfnis nach Beheimatung. Dieses Bedürfnis versucht auch, ein Gegengewicht gegen die von Winnicott (1974) beschriebenen frühkindlichen archaischen Daseins- bzw. Zerfallsängste ("Fear of breakdown") zu bilden (vgl. Durban, 2018, S. 46). Spätere Heimatvertreibungen stellen Retraumatisierungen des ursprünglichen Heimatverlusts dar. Vielleicht deswegen bezeichnet Freud (1933a, S. 62) das Unbewusste als «inneres Ausland», es ist das «ehemals Heimische, Altvertraute» (Freud, 1919h, S. 259). Winnicott (1988, S. $122 \mathrm{ff}$.) spricht ausdrücklich davon, dass die Seele erst im Laufe der (frühen) 
Entwicklung eine «Unterkunft» [lodgement] im Körper finden muss und mit Hilfe einer hinreichend guten Mitwelt zu «bewohnen» [indwelling] beginnt.

Wie jede Pflanze und jeder Baum nur zu seiner angemessenen Grösse heranwachsen kann, wenn sie fest in der Erde verwurzelt sind, ist es auch mit dem Menschen. Zu seinem vollen Menschsein benötigt er nach den Untersuchungen der Bindungsforschung und der Entwicklungspsychologie eine feste innere Verwurzelung oder Verankerung. Johann Wolfgang von Goethe wird der Satz zugeschrieben: «Wenn Kinder klein sind, gib ihnen Wurzeln, wenn sie gross sind, verleih ihnen Flügel».

So steht der Mensch lebenslang auch innerlich zwischen seinem Bedürfnis nach Sesshaftigkeit (mit dem Extrem des Entwicklungsstillstandes), dem «Heimweh» einerseits und dem Bedürfnis nach Nomadentum, Aufbruch und Abenteuer (mit dem Extrem der Selbstauflösung), dem «Fernweh» andererseits.

Fortan bewegt sich der Mensch in der Spannung zwischen seiner regressiven Sehnsucht nach Heimkehr in den als paradiesisch und vollkommen sicher phantasierten Mutterleib (Chasseguet-Smirgel 1988, 91 ff.), seinem «Konservativismus» und seiner progressiven Sehnsucht nach Freiheit, Lebendigkeit und Neuem. Diese ist aber grundsätzlich mit einem gewissen Unbehaustsein, mit Nicht-Wissen und mit Unsicherheit verknüpft. Sie können besser ausgehalten werden, wenn aus den vorangegangenen guten Erfahrungen verinnerlichte innere «Re-präsentanzen» von versichernden "guten Objekten» und von "guten Selbstanteilen» vorhanden sind.

Das Bedürfnis, festen Boden unter den Füssen zu haben, eine sichere Bleibe zu besitzen, uns wirklich zu Hause fühlen zu können, scheint in diesem Sinne ein sehr archaisches zu sein. Womöglich findet auch das Bedürfnis nach einem «Eigenheim» hier seine unbewusste Quelle? Aber auch das Gegenbedürfnis, zum Aufbrechen, unser «Traum vom Fliegen», das Bedürfnis nach Urlaub, der Wunsch, «Spielräume» zu entdecken, Neues zu erfahren, Abenteuer zu erleben, aber auch «Luftschlösser zu bauen», «aus sich herauszugehen» scheint ebenso tief in der menschlichen Seele verwurzelt.

Michael Balint (1960) hat das Verhältnis zwischen dem Heimweh und dem Fernweh mit den Begriffen «Oknophilie» [Liebe zur Nähe] und «Philobatie» [Liebe zur Weite] bezeichnet. Die dem entsprechenden krankhaften seelischen Raumstörungen sind: «Klaustrophobie»und«Agoraphobie».

Die Spannung zwischen beiden bildet sich auch ab in der lebenslänglichen Polarität zwischen Bindungsbedürfnis und Individuationsstreben und zwischen den Passivitätswünschen und den Aktivitätstendenzen des Menschen. Juan Carlos Rey (1979, S. 255) hat diese Spannung trefflich als das «klaustrophobisch-agorapho- 
bische Dilemma» benannt. Nach Balint (1960) ist jedes Aufbrechen in der Regel psychisch mit einer gewissen «Angstlust» [thrill] verknüpft.

Innerhalb und ausserhalb des Hauses oder der Wohnung Höhlen zu bauen, gehört zu den beliebten Kinderspielen und «Zelten» zu beliebten Pubertätserfahrungen. Später kann zum Beispiel das eigene Zimmer, die «eigenen vierWände» zugleich einen Schutzraum bilden, ebenso aber eine Abgrenzung nach aussen ermöglichen. Auch Matthias Hirsch (2006, S. 12) betont die Doppelfunktion und Ambiguität des Hauses, in das man sich einerseits zurückziehen und abschotten kann oder andererseits durch ein "offenes Haus» gerade sozialen Kontakt zur Mitwelt herstellen kann. Dieter Funke (2006, S. 85) schreibt entsprechend von der «grenzsetzenden und grenzöffnenden Funktion» des Hauses. Das Haus kann also zugleich ein Ort der Trennung und ein Ort der Verbindung sein (vgl. Hirsch, 2006, S.81). Insbesondere die Tür charakterisiert in besonderer Weise den Doppelcharakter dieses Sich-Abgrenzens und Sich-Öffnens (Funke, 2006, S. 40). Durch die Türe brechen wir aufund an der Türe kommen wir an. Die Journalistin Regine Igel (2005) formuliert im Anschluss an den Psychoanalytiker Uwe Langendorf (2004): «Heimat bedeutet also keine Höhle, die den Menschen abschottet, sondern eine Brücke zur Welt».

In einem Vortrag über «Frühstörungen» habe ich vor vielen Jahren (1992) vom «Niemandskind im Niemandsland» gesprochen. Aufgrund fehlender oder unzureichender emotionaler und/oder kognitiver Resonanz entsteht für solche Menschen am Beginn ihres Lebens im Innern keine Verwurzelung, keine Zugehörigkeit, kein «Heimatland», sondern eben ein «Niemandsland». Der israelische Kinderanalytiker Joshua Durban (2017) bezeichnet dies als “nowhereness".

\subsection{Anale Konflikte}

In den Konflikten der sog. «analen Phase», im 2./3. Lebensjahr wird entweder eine feste und stabile Heimatbasis des «Ichs» begründet, oder eine grundlegende Unsicherheit und Instabilität. Die in dieser Zeit besonders häufigen «Neins» des Kindes sind die ersten deutlicheren Manifestationen seines Ichs (vgl. Spitz, 1957). Die vielen «Neins» der Erwachsenen können durch ihren begrenzenden Charakter eine wichtige Hilfe für die Ich-Entwicklung und Strukturbildung darstellen. Neins im Übermass dagegen gefährden die Entwicklung einer stabilen und gesunden Ich-Basis. In der Körperlichkeit geht es vor allem um Festhalten und Loslassen (Ausscheiden), um erste Erfahrungen von Besitz («Identität») und Verlust von Eigenem und um «Reinheit» und Verschmutzungsfantasien über das 
Fremde. Beim analen Bestehen auf«Homogenität» wird das frühe Bedürfnis nach Ungetrenntsein wiederbelebt.

\subsection{Adoleszenz zwischen Entheimatung und Heimatsuche}

Die markanteste Aufbruchphase ist natürlich die Adoleszenz. Ein unerschütterliches, «nichtzerstörbares» (Winnicott) Elternhaus ist die Ausgangsbasis für explorative Aufbrüche und zugleich ein sicherer Rückkehrort (vgl. Kennedy, 2016, S. 812).

Der Psychoanalytiker Horst Eberhard Richter hat schon 1970 eine pathologische Form der Familie als «Festung» beschrieben. "My home is my castle", lautet eine britische Redensart. Sogar Erwachsene bauen am Strand «Sandburgen». Allerdings führt der Versuch eines übermässigen Geschützt- und Geborgenseins gegen Verunsicherungen zu Zwängen, zum Zuchthaus oder Gefängnis, und macht die Menschen darin krank. «Freiheit stirbt mit Sicherheit» soll Kurt Tucholsky (1934) gesagt haben. Vielleicht ist angesichts wachsender Umweltverseuchung und des Klimawandels die «Arche» - mit der man aufbrechen und ankommen kann - heutzutage ein angemesseneres Symbol als die «feste Burg»?

Ein Haus oder Heim kann uns bergen, Geborgenheit vermitteln («Herberge»). Im Haus kann aber auch Schlimmes «im Verborgenen», heimlich - hinter einer blendenden Fassade - geschehen, zum Beispiel familiäre seelische, sexuelle und andere Gewalt. Familientragödien und Seelenmorde spielen sich zumeist im Raum von Wohnung oder Haus ab.

Unser Leben ist durchgängig mit einer Menge an Unsicherheiten bestückt. Das Einzige, das sicher ist, ist - wie es Sigmund Freud in einem Brief (an Max Eitigon vom 05.01.1933; zit. n. Schur, 1972, S. 521) formulierte, dass «wir alle zum Tode verurteilt sind». Auch deshalb ist es uns wohl bewusst und unbewusst lebenslang so wichtig, festen Boden unter den Füssen zu spüren, vier stabile eigene Wände zu besitzen, ein festes Dach über dem Kopf, also ein «sicheres» Haus, eine Bleibe, eine Heimat. Zwischen unserer Geburt und unserem Tod befinden wir uns in einer "prinzipiellen Heimatlosigkeit», in der immer nur vorübergehende «Verortungen» möglich scheinen. Am Ende finden wir unsere letzte Behausung, unsere letzte «Ruhestätte» in einer Kiste, die in der «Mutter Erde» versenkt wird. Vollendet sich da unser «Aufbruch nach rückwärts»?

\section{$5 \quad$ Entheimatung}

Die Frage ist, ob in unseren postmodernen, «flüchtigen Zeiten» die Balance zwischen den Grundtendenzen Heimweh und Fernweh noch gewährleistet ist. 
In der Flüchtigen Moderne, wie der polnische Sozialwissenschaftler Zygmunt Baumann (2003) sie nennt, scheint es immer schwieriger, feste Wurzeln zu schlagen oder irgendwo anzukommen. Da gibt es einerseits die Millionen «Pendler», die ihr Heim für die Berufstätigkeit verlassen, und andererseits die Hunderttausende von «Fernbeziehungen», die unerträgliche «Liebe auf Distanz», wie es in einem neueren Schlagerlied heisst. Der postmoderne Mensch hat es unter diesen Umständen schwer, zur Ruhe zu kommen, Ruhelosigkeit ist das dominante Empfinden. Und das verstärkt vermutlich unbewusst das Bedürfnis nach Orten der Ruhe, nach Heimat.

Das Raumgefühl des heutigen Menschen und auch sein Zeitempfinden wird fortwährend destabilisiert und dekonstruiert, statt bestätigt und bekräftigt. Die modernen Verkehrsmittel erlauben eine derart beschleunigte Fort-Bewegung, dass bisweilen unsere Seele dem nicht nachzukommen vermag. Vielreisende wissen bisweilen am Morgen nicht, an welchem Ort der Welt sie gerade aufwachen. «In den Zeiten raschen Wechsels werden die Orte bedeutungsloser und auswechselbarer. Sie verlieren ihre Eigentümlichkeit, damit aber auch die Kraft, Menschen zu bergen» schreibt der Theologe Fulbert Steffensky (2003, S. 49; vgl. Langendorf, 2004, S. 210). Die zunehmende Beschleunigung, der der moderne Mensch ausgesetzt ist, erschwert oder verhindert gar seine Beheimatung. Und weiter: «Es gibt eine neue, fast omnipotente Gleichzeitigkeit, die den Menschen entheimatet und ortlos macht» (Steffensky, 2003, S. 50). Die (fast) grenzenlose Mobilität erschwert die sichere Orientierung und die Verortung in Zeit und Raum. «Der moderne Mensch tauscht Heimat gegen Welt ein», schreibt Jean Améry schon 1966 (1988, S. 75)! «Der Markt verlangt Räder. Aber der Mensch braucht Wurzeln», formuliert der Familienforscher Dirk Weissleder (zit. n. Sell, 2013, S. 12).

Aktuell erleben wir im Rahmen der Corona-Krise (Auchter, 2020) problematisch das Gegenteil von Heimatverlust, nämlich vielerorts auf der Welt den Zwang in der Heimat zu bleiben, zu Hause eingesperrt zu sein. Das coronabedingte Fixiertsein (Lockdown) an das Zuhause stellt das Gleichgewicht zwischen Fernweh und Heimweh massiv in Frage. Die Abenteuerlust (Urlaub im Ausland) bezahlt die Gesellschaft mit steigenden Infektionszahlen! Seitdem boomt der Markt mit dem «Haus auf Rädern», den Wohnmobilen.

\section{Heimatverlust}

Neben der seelischen Heimatlosigkeit in der postmodernen Welt ist die Gegenwärtigkeit durch vielfältige Flüchtlings- und Migrationsbewegungen charakterisiert, die die globale Verunsicherung und Beängstigung und die fundamentale Unsicherheit der Existenz, das «Geworfensein in die Welt» Martin Heideggers nur 
verstärken. Wenn wir den Gedanken von Roger Kennedy (2016, S. 805) ernst nehmen, dass für uns das Empfinden von fundamentaler Bedeutung ist, «ein Zuhause zu haben, als Grundlage unseres Seins, als Ort, den wir brauchen, um uns geborgen zu fühlen», dann wird klar, welche Wirkung erzwungene Heimatverluste durch Krieg, Gewaltakte, Hunger und Vertreibung besitzen.

Für die Flüchtlinge ist ja in der Regel nicht nur ihr äusseres Zuhause, ihre Heimat verlorenen gegangen oder zerstört worden, sondern gleichermassen ist das verbunden mit dem mehr oder weniger traumatischen Verlust der inneren Heimat, des «psychischen Zuhauses», einer stabilen seelischen Struktur.

Wenn das Gefühl des «Heimischseins» vor allem aus Beziehungen erwächst, dann wird auch verständlich, welche Wirkung der mit der Flucht in der Regel verbundene Beziehungsverlust, die Trennung von Familienangehörigen (ich denke da vor allem an die vielen unbegleiteten minderjährigen Flüchtlinge) und dem sozialen Umfeld hat. Für die meisten Menschen bedeutet der Heimatverlust eine mehr oder minder ausgeprägte «Traumatisierung» (vgl. Langendorf, 2004).

Sinnbildlich für den Übergang zwischen alter und neuer Heimat sind vielleicht die «Container», in denen viele heutige Flüchtlinge zunächst untergebracht sind. Die Container bergen und schützen einerseits, anderseits sind sie (nur) zeitlich begrenzte Gefässe, die jedoch im besten Fall Veränderungen möglich machen und begleiten.

Neben dem endgültigen Heimatverlust finden wir auch den vorübergehenden der «Emigration». Dabei dominiert die Erwartung und Hoffnung auf eine Rückkehr in die verlassene Heimat.

Darüberhinaus haben wir es seit längerem mit einem (Heimat)Verlust traditioneller Zugehörigkeiten und Verbundenheiten (Parteien, Kirchen, Gewerkschaften) zu tun. Da der Mensch aber nicht auf Bindungen zur Stabilisierung seiner Identität verzichten kann, sucht er zumindest unbewusst nach Ersatz. Schon im Begriff der «Identitären» steckt die übermässige Betonung der gefährdeten Identität drin. Diese muss abgesichert werden durch Abgrenzung, Abwertung und Feindseligkeit gegenüber anderen «Nicht-Ichs» und fremden Identitäten, Homogenität auf Kosten von Heterogenität! Gudrun Brockhaus (2018, S. 273) spricht in einer jüngeren Arbeit diesbezüglich vom gesellschaftlichen respektive politischen «Klimawandel».

\section{Die Fähigkeit, «neue Heimaten» zu schaffen}

Neben seinem - vielleicht unstillbaren - regressiven Bedürfnis nach der«alten Heimat» besitzt der Mensch aber auch eine gewisse Fähigkeit «neue Heimaten» zu schaffen, zur «Heimatbildung» (Türcke, 2006, S. 69). Sverre Varvin (2016, S. 172) 
bringt das mit «Hoffnung» zusammen. Rainer Gross (2019, S. 139) bezeichnet die Entwicklung eines Heimatgefühls als «komplexe Entwicklungsleistung». Ohne diese Fähigkeit wäre Weiterentwicklung überhaupt nicht möglich. Denn Entwicklung besteht in einem ständigen «Abschied und Neubeginn» (Balint, 1932).

So wie das Subjekt am Lebensbeginn seine persönliche Heimat aus seinem beziehungsmässigen Nahraum, seiner sozialen, materiellen und kulturellen Lebensmitwelt konstituiert, so ist das in aller Begrenztheit (vgl. Améry, 1988, S. 67) auch im späteren Leben möglich. Menschen, die dazu nicht in der Lage sind oder dem nicht gewachsen sind, werden zu pathologischen Lösungen greifen.

\section{Zur Psycho-sozio-Pathologie von «Heimat»}

Das Konzept «Heimat» ist tief in der Lebensgeschichte des Individuums, tief in seiner Emotionalität und tief in seinem Unbewussten verankert. Dadurch bietet es Interessierten, zum Beispiel Rechtspopulisten eine Fülle von Angriffsund Manipulationsmöglichkeiten. Da Heimat im Unbewussten mit Sicherheit und Geborgenheit assoziiert ist und entsprechend das Fremde mit Bedrohlichkeit und Angst (Auchter, 1993; 2016), nutzen Rechtspopulisten diese Psychodynamik, um Heimat für sich zu instrumentalisieren. Als Gegenbild bauen sie Offenheit, Multikulturalität und Flüchtlinge als Angstkulisse auf, um dagegen Heimat, Volk und Vaterland als idealen Ort der Sicherheit hochzustilisieren.

Wer keine hinreichend gute, ebenso stabile wie flexible innere Heimat sein eigen nennt, der ist gezwungen, auf einer rigiden äusseren Heimat zu bestehen. Anstelle von Brücken werden Gräben, Stacheldrahtzäune und Mauern errichtet. Das Ertragenkönnen von Ambivalenzspannungen vermindert derartige Spaltungstendenzen und die innere Notwendigkeit von solchen Feindbildungen. Der Freiburger Psychoanalytiker Gehad Mazarweh, als Palästinenser selbst einst von den Israelis aus seinem Heimatland vertrieben, meint in einem Interview knapp: «Wer Mauern braucht, hat Angst»!

Als spezifische lebensgeschichtliche Blaupause für die «Heimatpathologie» ist wie schon erwähnt die Familie von Bedeutung. Im kindlichen Entwicklungsverlauf können zu einem bestimmten Zeitpunkt alle die fremd werden, die nicht zur Familie gehören (Erdheim, 1988). Anna Freud (1948) sieht darin eine Folge des unausgehaltenen Ambivalenzkonflikts zwischen Liebe und Hass gegenüber Eltern und Geschwistern. Indem alle missgünstigen Regungen von der Familie weg auf die Aussenwelt gelenkt und in sie projiziert werden (vgl. Richter, 1970). In einem solchen Fall wird die Familie zum «nur guten» Objekt, «von dem sich das Individuum gar nicht trennen kann, während die Kultur [oder die Mitwelt] als 
das gefährliche Böse erscheint» (Erdheim, 1988, S. 239). Ob die äussere Heimat z. B. durch Fremde als bedroht erlebt wird oder nicht, hängt von der Stärke und Sicherheit der inneren Heimat ab.

Problematisch wird das Konzept «Heimat», wenn um sie herum eine hermetische Grenze gezogen wird. Wenn also Heimat nicht mit Inklusion und Integration einhergeht, sondern durch Exklusion abgesichert werden muss. Wenn Heimat ausschliesslich reserviert ist für «Einheimische».

Eine überstarke Abgrenzung macht Heimat zu einem destruktiven Kampfbegriff anstelle einer «Brücke zurWelt» (Igel, 2005). «Entscheidend für einen vernünftigen Umgang mit Heimat ist, dass ihre Überschätzung sich zur Schätzung ermässigt» (Türcke, 2006, S. 29). Freud (1939a, S. 197) schreibt: „Das Gemeinschaftsgefühl der Massen braucht zu seiner Ergänzung die Feindseligkeit gegen eine aussenstehende Minderzahl». Dieser Gedanke macht noch einmal deutlich, wie wichtig eine stabile innere Heimat ist, um derartigen Tendenzen entgegenzuwirken und eine «Toleranz für Differenz» zu entwickeln!

\section{Literatur}

Améry, J. ([1966] 1988). Wieviel Heimat braucht der Mensch? In J. Améry, Jenseits von Schuld und Sühne (S. 59-80). dtv/Klett-Cotta.

Auchter, T. (2016). Das Selbst und das Fremde. Zur Psychoanalyse von Fremdenfeindlichkeit und Fundamentalismus. Psyche, 70, 856-880.

Auchter, T. (2013). Äussere Realität, innere Realität und der potential space bei DonaldW. Winnicott. In T. Hartung \& L. V. Strauss (Hrsg.), Tauchgänge. Psychoanalyse der äusseren und inneren Realität (S. 123-145). Vandenhoeck \& Ruprecht.

Auchter, T. (2004). Zur Psychoanalyse des Möglichkeitsraumes «Potential Space». Freie Assoziation, 7, 37-58.

Auchter, T. (2000). Das Konzept des Haltens und seine Bedeutung für die allgemeine und psychotherapeutische Entwicklung. Zeitschrift für Individualpsychologie, 25, 88-99.

Auchter, T. (1993). Die seelische Krankheit «Fremdenfeindlichkeit». In U. Streeck (Hrsg.), Das Fremde in der Psychoanalyse (S. 225-234). Pfeiffer.

Auchter, T. (1992).Das Niemandskind im Niemandsland. Zur Phänomenologie, Genese, Psychodynamik und psychoanalytischen Behandlung von Frühstörungen. Unveröffentlichtes Manuskript.

Balint, M. ([1968] 1970).Therapeutische Aspekte der Regression. Klett. 
Balint, M. (1960). Angstlust und Regression. Beitrag zur psychologischen Typenlehre. Klett.

Balint, M. (1932). Charakteranalyse und Neubeginn. In M. Balint ([1965] 1966). Die Urformen der Liebe und die Technik der Psychoanalyse (S. 187-202). Klett. Baumann, Z. (2003). Flüchtige Moderne. Suhrkamp.

Brockhaus, G. (2018). Erosion von Struktur und Gewissheit. Zeitschrift für Gruppenpsychotherapie und Gruppendynamik, 54, 269-292.

Chasseguet-Smirgel, J. (1988). Zwei Bäume im Garten. Zur psychischen Bedeutung der Vater- und Mutter-Bilder. Verlag Internationale Psychoanalyse.

Durban, J. (2018). Heimat, Heimatlosigkeit und Nirgendwo-Sein in der frühen Kindheit. In M. Johne, G. Allert et al. (Hrsg.), Veränderung im psychoanalytischen Prozess - Entwicklung und Grenzen (S. 273-295).Tagungsband der DPV-Herbsttagung 2017.

Durban, J. (2017). Home, homelessness and nowherenes in early infancy. Journal of Child Psychotherapy, 43, 175-191.

Erdheim, M. (2016). Die Unvermeidlichkeit der Heimatlosigkeit und die Fähigkeit, neue Heimaten zu schaffen. In I. Focke \& G. Salzmann (Hrsg.), Heimatlos. Psychoanalytische Erkundungen (S. 107-116). DPG Tagungsband.

Erdheim, M. (1988). Die Repräsentanz des Fremden. In M. Erdheim, Die Psychoanalyse und das Unbewusste in der Kultur (S. 237-251). Suhrkamp.

Focke, I. \& Salzmann, G. (Hrsg.). (2016). Heimatlos. Psychoanalytische Erkundungen. DPG Tagungsband.

Freud, S. (1939a).Der Mann Moses und die monotheistische Religion. GW XVI, S. $103-246$.

Freud, S. (1933a [1932]). Neue Folge der Vorlesungen zur Einführung in die Psychoanalyse. GWXV.

Freud, S. (1930a). Das Unbehagen in der Kultur. GW XIV, S. 419-506.

Freud, S. (1926d). Hemmung, Symptom und Angst. GW XIV, S. 111-205.

Freud, S. (1919h). Das Unheimliche. GW XII, S. 229-268.

Funke, D. (2006). Die dritte Haut. Psychoanalyse des Wohnens. Psychosozial Verlag.

Gross, R. (2019). Heimat. Gemischte Gefühle. Vandenhoeck \& Ruprecht.

Hermanns, L. M. (Hrsg.). (1995). Spaltungen in der Geschichte der Psychanalyse. edition diskord.

Hirsch, M. (Hrsg.). (2006). Das Haus. Symbol für Leben und Tod, Freiheit und Abhängigkeit. Psychosozial Verlag.

Hoven-Buchholz, K. (2016). Heimat? - Los! In I. Focke \& G. Salzmann (Hrsg.), Heimatlos. Psychoanalytische Erkundungen (S. 138-154). DPG Tagungsband. 
Igel, R. (2005). Wenn du all das verlässt, was zu dir gehört, verlässt du fast dich selbst. In: www.freitag.de/autoren/der-freitag/wenn-du-all-das-verlasstwas-zu-dir-gehort-verlasst-du-fast-dich-selbst. [22.12.2020].

Janus, L. (2016). Die Urheimat vor der Geburt als Tiefendimension von Heimat. In I. Focke \& G. Salzmann (Hrsg.), Heimatlos. Psychoanalytische Erkundungen (S. 72-95). DPG Tagungsband.

Kennedy, R. (2016). Furcht vor Fremden: Wessen Zuhause ist das hier? Psyche, 70, 805-824.

Langendorf, U. (2004). Heimatvertreibung - das stille Trauma. Analytische Psychologie 35, S. 206-223.

Leszczynska-Koenen, A. (2016). Heimat ist kein Ort. In I. Focke \& G. Salzmann (Hrsg.), Heimatlos. Psychoanalytische Erkundungen (S. 214-228). DPG Tagungsband.

Mahler, M., Pine, F. \& Bergmann, A. ([1975] 1978). Die psychische Geburt des Menschen. Symbiose und Individuation. Fischer.

Messer, P. (2016). Liebe ist Heimweh. In I. Focke \& G. Salzmann (Hrsg.), Heimatlos. Psychoanalytische Erkundungen (S. 96-106). DPG Tagungsband.

Morgenstern, C. (1918). Stufen. Eine Entwicklung in Aphorismen und TagebuchNotizen. Piper.

Pavlović, M. (2016). Die Heimat(losigkeit in) der Psychoanalyse. In I. Focke \& G. Salzmann (Hrsg.), Heimatlos. Psychoanalytische Erkundungen (S. 155170). DPG Tagungsband.

Rey, J. C. (1979). Schizoide Phänomene im Borderline-Syndrom. In E. B. Spillius, (Hrsg.), Melanie Klein heute. Bd. 1. Beiträge zur Theorie (S. 253-287). Verlag Internationale Psychoanalyse.

Richter, H. E. (1970). Patient Familie. Entstehung, Struktur und Therapie von Konflikten in Ehe und Familie. Rowohlt.

Schmoll, F. (2016). Heimat, eine Gebrauchsgeschichte zwischen Fürsorge und Verbrechen. In I. Focke \& G. Salzmann (Hrsg.), Heimatlos. Psychoanalytische Erkundungen. (S. 15-23). DPG Tagungsband.

Schur, M. (1973). Sigmund Freud. Leben und Sterben. Suhrkamp.

Sell, D. (2013). Alle an einen Tisch. Ein Gespräch mit dem Familienforscher Dirk Weissleder. Publik-Forum Extra. September 2013, Heimat, 12-13.

Sennett, R. ([1998] 1999). Der flexible Mensch. Die Kultur des neuen Kapitalismus. Berlin Verlag.

Spitz, R. (1957). Nein und Ja-Die Ursprünge der menschlichen Kommunikation. Klett. 
Steffensky, F. (2003). Ganzheit im Fragment. Heil und Heilung in unserer Zeit. In H. Egner (Hrsg.), Heilung und Heil. Begegnung - Verantwortung - Interkultureller Dialog (S. 40-59). Patmos.

Türcke, C. (2006). Heimat. Eine Rehabilitierung. Zu Klampen Verlag.

Varvin, S. (2016). Exil und Heimatlosigkeit im Schatten extremer Traumatisierung. In I. Focke \& G. Salzmann (Hrsg.), Heimatlos. Psychoanalytische Erkundungen (S. 171-186). DPG Tagungsband.

Winnicott, D. W. (1988). Human Nature. Free Association.

Winnicott, D. W. (1986). Home is where we start from. Penguin Books.

Winnicott, D. W. (1974). Fear of Breakdown. International Review Of Psychoanalysis, 1, 103-107.

\section{Angaben zum Autor}

Thomas Auchter, Dipl. Psych., 1948, ist als Psychoanalytiker (DPV/IPA/ DGPT) in freier Praxis niedergelassen in Aachen, Lehranalytiker und Dozent am Institut der Psychoanalytischen Arbeitsgemeinschaft Köln-Düsseldorf (DPV). Neben umfangreicher Vortragstätigkeit zahlreiche Publikationen, vor allem auch im Bereich der angewandten Psychoanalyse. 1999 mit seiner Düsseldorfer Kollegin Laura Viviana Strauss Veröffentlichung von Kleines Wörterbuch der Psychoanalyse. 2012 im Psychosozial Verlag: Brennende Zeiten. Zur Psychoanalyse sozialer und politischer Konflikte; 2019: Trauer. 